\title{
LYMPHANGIOMA OF A RARE SITE AND AT RARER AGE OF PRESENTATION: A CASE REPORT
}

\section{Rijhsinghani Archana N*}

Junior consultant, Department of Histopathology, SRL Diagnostics Dr Avinash Phadke Labs, Shivaji Park, Dadar, Mumbai *Corresponding Author

ABSTRACT Lymphangiomas are benign lesions characterized by the proliferation of lymphatic spaces, primarily diagnosed in children. It most often occur in the head, neck, axilla, or groin. Infrequently, intra-abdominal lymphangiomas are diagnosed in adults, primarily and age of presentation.

\section{KEYWORDS : Lymphangioma, intra-abdominal, mesentry, adult}

\section{Introduction}

Lymphangiomas most often occur in the head, neck, axilla, or groin of young children. Occasionally, the tumour is also discovered in adults in various other anatomical sites. Approximately $95 \%$ of lymphangiomas are found in the neck and axilla and the other $5 \%$ occur in the mediastinum and abdominal cavity, including the mesentery, retroperitoneum and bones. Intra-abdominal lymph-angiomas (fewer than $5 \%$ ) have been reported in the mesentery, gastrointestinal tract, spleen, liver and pancreas. [1]

Intra-abdominal cystic lymphangiomas most commonly occur in children younger than 10 years. Ninety percent of children with these lesions receive a diagnosis before age 2 years. Rarely, the lesion presents in adolescence or adulthood. Within the gastrointestinal tract, the small bowel mesentery is the most common site involved, followed by retroperitoneal sites.

They are most frequently found incidentally. Occasionally, they may cause abdominal pain, vomiting, and/or alterations in bowel habits due to intestinal compression, obstruction, and/or intussusception. Although acute complaints tend to occur in children, adults with these lesions tend to experience more chronic symptoms.[2]

Abdominal cystic lymphangiomas are more frequent in boys (5:2) with a mean age at presentation of 2 years. [3]

Ultrasonography is very sensitive at detecting cystic masses and can be used to better define them. Cystic lymphangiomas may appear as multilobular masses with internal septations and anechoic fluid. The identification of intracystic and/or intercystic echogenic septae within the lesion is an important element of the diagnosis. One limitation of ultrasonography in defining these lesions is that their relatively large size may interfere with the evaluation of their borders and sites of origin. CT scans can be used to better define the location and extension of the cystic masses. This imaging modality can also determine the attenuation coefficient of the cystic fluid, defining it as chylous fluid, blood, or pus. MRI is more sensitive and specific for the diagnosis of hemorrhagic complications but otherwise offers little information not already provided by the combination of ultrasonography and CT. Arteriography, lymphangiography, and radionuclide scans are rarely useful in the evaluation of intra-abdominal cystic lymphangiomas. Surgical resection is the definitive treatment for abdominal cystic lymphangiomas. [2]

We report a case of mesenteric lymphagioma with involvement of ileal wall in an older male patient which was treated surgically. We are presenting this case due to rare location of this tumour with rarer age of presentation.
A 42-year-old male presented with vague abdominal pain of seven months duration. Clinical examination did not reveal any palpable mass. Radiology was suggestive of multilocular cystic mass in the mesentry involving wall of ileum.

Surgical resection was done and we received $9.5 \mathrm{~cm}$ long segment of ileum. The attached part of mesentry was externally bosselated (Figure 1). The cut surface revealed multilocular cystic tumour in mesentry extending to the wall of ileum measuring $5.5 \times 5 \times 4 \mathrm{cms}$ (Figure 2). Foci of haemorrhage were seen. Both the resection margins were clear. Microscopic examination revealed thin walled ectatic vascular channels lined by flat bland looking endothelial cells with wall showing smooth muscle bundles and lymphoid aggregates at places. Lesion involved ileal mesentry and extended to involve wall of ileum (Figure $3,4,5$ ). The mucosa of ileum was eroded at places. Final diagnosis given was mesentric lymphangioma with involvement of ileal wall.
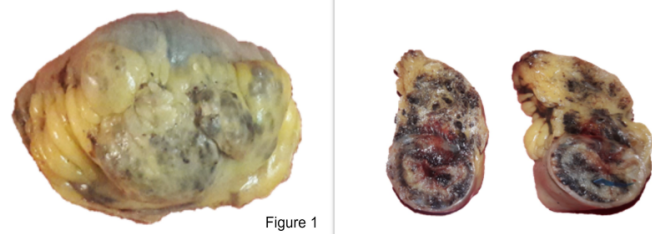

Figure 2

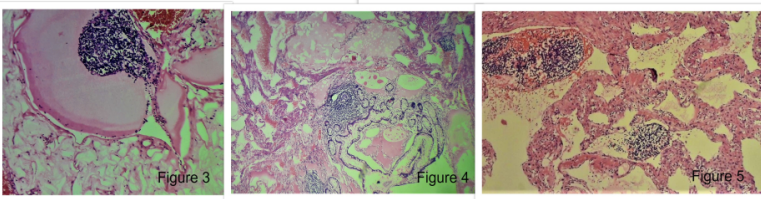

Figure 1 and 2: Gross -Ileum showing multilocular cystic tumour in mesentry extending to the wall of ileum.

Figure 3: Microscopy (HE x100)- Thin walled ectatic vascular channels surrounded by lymphoid aggregates amidst mesenteric adipose tissue.

Figure 4: Microscopy (HE x40)- Lymphangioma extending into intestinal wall.

Figure 5: Microscopy (HE x 40)- Thin walled ectatic vascular channels lined by flat bland looking endothelial cells with wall showing smooth muscle bundles and lymphoid aggregates.

\section{DISCUSSION}

Lymphangioma is a mass-forming lesion characterized by numerous thin-walled lymphatic spaces and usually manifests in the first few years of life. The common sites are the head, neck, and axillary regions. Other locations such as the abdominal or mediastinal cavity are rare, accounting for approximately $5 \%$ of lymphangiomas. Among these, lymphangioma of the small-bowel mesentery has been described in less than $1 \%$ of lymphangiomas. Lymphangioma appears to result from congenital malformation of lymphatic 
vessels rather than a true lymphatic tumor. The former causes sequestration of lymphatic vessels during the embryonic period. However, some data suggest that inflammation, abdominal trauma, abdominal surgery, radiation, or lymphatic obstruction may play a role in the genesis as a tumor. [4]

The differential diagnosis of an intra-abdominal cystic mass involves enteric duplication cysts, enteric cysts, mesothelial cysts, pancreatic pseudocysts, non-pancreatic pseudocysts, cystic mesotheliomas, cystic spindle cell tumors, and cystic teratomas. [5]

Ultrasonography and CT are considered as the most appropriate radio-diagnostic modalities to evaluate mesenteric lymphangioma, although ultrasonography is usually sufficient. Ultrasonography can differentiate the lymphangiomas from simple mesenteric cyst, because the fluid contents in lymphangiomas are usually homogeneous, and attenuation values may range between those of fat and fluid. On CT scans, mesenteric lymphangiomas appear as uni- or multilocular masses containing septa of variable thickness; enhancement of the wall is revealed by contrast medium.[6]

Kim et al. presented a modified classification system for mesenteric lymphangiomas consisting of four groups: group 1, mesenteric lymphangiomas involving the intestinal wall; group 2, pedicled mesenteric lymphangiomas with no relationship to the mesenteric vessels; group 3, sessile mesenteric lymphagiomas located in the mesenteric boundaries near the mesenteric vessels; and group 4, multicentric and diffusely infiltrated mesenteric lymphangiomas. Among the 25 cases in Kim's report, 14 were classified into group 1 , and all of the lesions were excised with segmental resection of the intestine. [7] Our case can also be classified into group 1 of the above said classification and was surgically excised.

Lymphangiomas are traditionally classified into three histologic types: capillary (simple), cavernous, and cystic. The capillary type usually originates in the skin and consists of uniform small thin-walled lymphatic spaces. The cavernous type is composed of various sizes of dilated lymphatic spaces associated with lymphoid stroma and shows a connection with the adjacent normal lymphatic spaces. The cystic type consists of dilated lymphatic spaces of various sizes associated with collagen and smooth-muscle bundles in the stroma but lacks connection to the adjacent normal lymphatic spaces. Cystic lymphangioma findings are similar to cavernous lymphangioma findings in that dilated lymphatic spaces of variable size are seen in both.[1]

Surgical resection is the definitive treatment for abdominal cystic lymphangiomas. Depending on the extent and location of the cysts, bowel resection may be required. Complete en bloc resections usually result in excellent outcomes and prognoses for patients. A $10 \%$ recurrence rate has been described in patients who have undergone incomplete resections. In children, ultrasonography is the preferred imaging modality for postoperative surveillance. There are no published guidelines regarding the frequency at which surveillance ultrasounds should be performed.[2]

Ours was a case of cystic type of mesenteric lymphangioma presenting at 42 years of age, group 1 of Kim's classification, with no other complications treated well with surgical excision and there is no recurrence yet.

\section{CONCLUSION}

Abdominal lymphangiomas usually affects infants and young children, which may be detected incidentally or present with acute life-threatening abdominal obstruction. However, this diagnosis should also be considered in older patients having intraabdominal cystic mass. A total surgical excision seems to be the best therapeutic option in order to minimize the risk of recurrence.

\section{Acknowlegement}

The author would like to thank SRL Diagnostics Dr Avinash Phadke Labs.

\section{Financial support and sponsorship} Nil.

\section{Conflicts of interests}

There are no conflics of interest.

\section{REFERENCES}

1. Ignjatovic, I., Milosavljevic, V., Tadic, B., Grubor, N., \& Matic, S. Lymphangioma of the Small Intestine Case Report and Review of the Literature. Ser J Exp Clin Res 2019;20(4):357-360.

2. Ajay Rana, Philip J. Katzman, Walter Pegoli, Cary Qualia. An Unusual Cause of Abdominal Pain: Duodenal Cystic Lymphangioma. Gastroenterology \& Hepatology. 2013:9(3):192-196.

3. Konen O, Rathaus V, Dlugy E, et al. Childhood abdominal cystic lymphangioma. Pediatr Radiol 2002;32(2):88-94.

4. Suthiwartnarueput W, Kiatipunsodsai S, Kwankua A, Chaumrattanakul U. Lymphangioma of the small bowel mesentery: a case report and review of the literature. World J Gastroenterol 2012;18(43):6328-32.

5. Ömer Katı, Sükrü Güngör, Yasar Kandu. Mesenteric cystic lymphangioma: Case report. Journal of Pediatric Surgery Case Reports 2018;35:26-28.

6. Kumar B, Bhatnagar A, Upadhyaya VD, Gangopadhyay AN. Small Intestinal Lymphangioma Presenting as an Acute Abdomen with Relevant Review of Literature. J Clin Diagn Res. 2017 Jun; 1 1(6):PD01-PD02.

7. Kim SH, Kim HY, Lee C, Min HS, Jung SE. Clinical features of mesenteric lymphatic malformation in children. J Pediatr Surg. 2016;51:582-7. 